Vol 7 / Issue 2 / March-April 2017

Journal of

International Society of

Preventive \&

Community Dentistry

Publication of International Society of Preventive and Community Dentistry
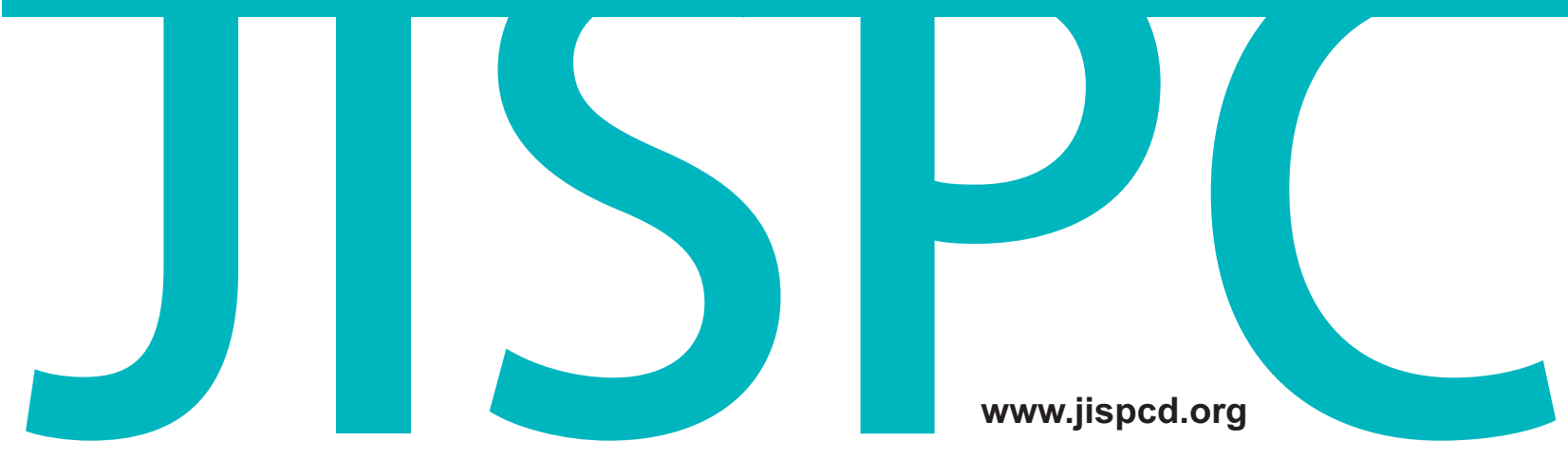

www.jispcd.org 


\section{Evaluation of Temporomandibular Disorders before and after Orthognathic Surgery: Therapeutic Considerations on a Sample of} 76 Patients

Carlo di Paolo, Giorgio Pompa ${ }^{1}$, Paolo Arangio², Anna di Nunno, Stefano Di Carlo ${ }^{1}$, Daniele Rosella ${ }^{1}$, Piero Papi ${ }^{1}$,
Piero Cascone ${ }^{2}$

Gnathology Unit, 'Implant

Prosthetic Unit, ${ }^{2}$ Cranio

Maxillofacial Unit,

Department of Oral and

Maxillo Facial Science,

"Sapienza" University of

Rome, Rome, Italy
Received : 16-10-16.

Accepted : 01-03-17.

Published : 29-03-17.
Objectives: Temporomandibular disorders may be associated with dental and facial malformations. The aim of this study is to record the prevalence of TMDs in patients scheduled for orthognathic surgery, reporting the development of TMDs and symptoms during the entire period of the treatment, and demonstrating the benefits of a team effort on this population.

Materials and Methods: Assessment of temporomandibular status was performed using the RDC/TMD criteria at T0 (prior to orthodontic therapy), T1 (3 months after the surgery), and T2 post-therapeutic cycle (6 to 12 months postoperatively). A total of 76 participants were included in the study; all the patients underwent surgical treatment: 12 had bilateral sagittal split osteotomy, 6 with condylar position devices; 64 had Le Fort I + bilateral sagittal split osteotomy, and 15 with condylar position devices. Results were evaluated with a paired-sample $t$-test and segmentation analysis.

Results: Forty-seven patients were affected by TMDs. At T0, 25 patients experienced TMJ pain, 27 had muscular pain, 31 suffered headaches, 42 had disc dislocation with reduction, and 5 were affected by disc dislocation without reduction. Thirty-five patients had occlusal signs of parafunctions, 8 reported tinnitus, and 7 dizziness. At T1, TMJ pain changed from $33.3 \%$ to $4.44 \%$, muscular pain changed from $35.5 \%$ to $11.1 \%$, headaches improved from $40 \%$ to $6.67 \%$, and disc dislocation from $55.2 \%$ to $17.7 \%$. Segmentation analysis highlighted improvement after therapy; 57 patients were considered recovered, 14 improved, none were considered stable, whereas 5 patients demonstrated some worsening, 3 of whom had not presented disc dislocation before surgery. At T2, 71 patients were considered completely recovered or improved.

Conclusions: Our data indicates beyond any doubt that both functional status and pain levels related to TMDs can be significantly improved with a multi-disciplinary approach. We concluded that surgeon's intervention need to be modified in the presence of presurgical TMDs.

Keywords: Dentoskeletral malformations, orthognathic surgery, temporomandibular disorders, TMDs therapy

\section{INTRODUCTION}

Dreviously, patients with dentoskeletal malformations 1 and head and neck tumors, who were scheduled for orthognathic surgery, routinely underwent

\begin{tabular}{|l|l|}
\hline \multicolumn{2}{c}{ Access this article online } \\
\hline Quick Response Code: & Website: www.jispcd.org \\
\hline
\end{tabular}

Address for correspondence: Dr. Piero Papi, Department of Oral and Maxillofacial Sciences, Sapienza University of Rome, Via Caserta 6, 00161 Roma, Italy. E-mail: papi.piero@gmail.com

This is an open access article distributed under the terms of the Creative Commons Attribution-NonCommercial-ShareAlike 3.0 License, which allows others to remix, tweak, and build upon the work non-commercially, as long as the author is credited and the new creations are licensed under the identical terms.

For reprints contact. reprints@medknow.com

How to cite this article: di Paolo C, Pompa G, Arangio P, di Nunno A, Di Carlo S, Rosella D, et al. Evaluation of temporomandibular disorders before and after orthognathic surgery: Therapeutic considerations on a sample of 76 patients. J Int Soc Prevent Communit Dent 2017;7:125-9. 
diagnostic evaluation to assess the functionality of temporomandibular joint (TMJ) and masticatory muscles. ${ }^{[1-3]}$ The therapy for patients with dentoskeletal malformations and temporomandibular disorders (TMDs) may be a demanding challenge for clinicians; it is not only important to correct skeletal malformation, but also to reduce arthralgia, muscular pain, and TMJ

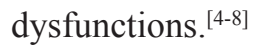

In the early 2000's, Pakula ${ }^{[9]}$ and Dervis, ${ }^{[10]}$ stated that functional TMJ status may be significantly improved and pain levels reduced with orthognathic surgery. On the other hand, Wolford ${ }^{[11]}$ asserted that patients with pre-existing TMJ dysfunction undergoing orthognathic surgery are likely to have significant worsening of the TMJ dysfunction postsurgery. According to Wolford, TMDs must be closely evaluated, treated if necessary, and monitored in the orthognathic patient. Aoyama in $2005^{[12]}$ stated that the surgical correction of dentoskeletal malformations may affect TMJ dysfunction symptoms. Abrahamsson in 2007, ${ }^{[13]}$ in order to answer the question regarding the effects of orthognathic surgery on TMDs, published a systematic review of the literature. In the paper, the authors highlighted the current difficulty to provide conclusive answers on this topic because of the unreliability of the studies emerged from the analysis of the literature, and stated the need for further studies. The same authors published in $2009^{[14]}$ a study that analyzed a consecutive series of patients referred for orthognathic surgery and compared them with a control group. They concluded that patients who were to be treated with orthognathic surgery had more signs and symptoms of TMDs.

To clarify these relationships, the aims of this study were to document the presence of TMDs in patients scheduled for orthognathic surgery and to record the development of TMDs and symptoms during the entire treatment period.

\section{Materials and Methiods}

The patient population comprised a consecutive series of patients with dentoskeletal malformations referring to the Department of Oral and Maxillofacial Sciences, Sapienza

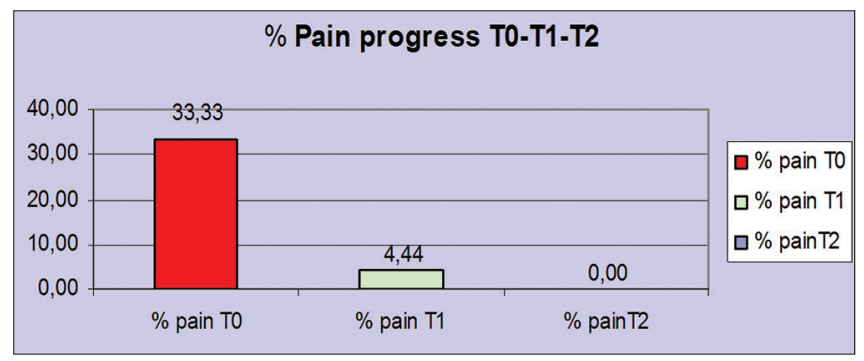

Figure 1: TMJ pain progression during treatment
University of Rome, from January 2003 to January 2006. The initial study population comprised 1528 patients. A total of 460 patients were excluded because they lived too far from Rome; 1168 were examined and 1047 were barred according to strict inclusion and exclusion criteria. Inclusion criteria were patients with dentoskeletral malformations and surgical treatment by bilateral sagittal split osteotomy (BSSO) of the mandible or le Fort I plus BSSO. Exclusion criteria included presence of systemic joint or muscular disorders, presence of organic lesion, or traumatic outcome of TMJ or in any other part of the maxillofacial district (TMJ ankylosis, etc.). Patients who reported previous orthognathic surgical or TMD therapy or those suffering from psychiatric disorders were also excluded. The study protocol included an initial maxillofacial assessment. Second, patients were examined by an interdisciplinary team (surgeon, radiologist, orthodontist, prosthodontist, and gnathologist of the Dental School of the same University) to assess the status of the TMJ. The examiners in each area of specialization remained the same.

Assessment of temporomandibular status was performed using the RDC/TMD criteria at T0 (prior to the orthodontic therapy), T1 (3 months after the surgery), and T2 post-therapeutic cycle (6-12 months postoperatively).

At T0, the following data were registered: TMD frequency, type and prevalence, percentage and diffusion of the joints and muscular pain, ${ }^{[13,14]}$ as well as pain intensity levels in the joints using a severity scale related to the visual analog scale (VAS) scale. The same method of evaluation was used for headache. ${ }^{[15]}$

We monitored the progression of the TMDs during the orthodontic therapy and assigned a value to the progression at the end of this phase before surgery [Figures 1 and 2].

All patients underwent surgical treatment: 12 (15.7\%) had bilateral sagittal split osteotomy (BSSO), 6 with condylar position devices; $64(84.2 \%)$ had Le Fort I + BSSO, 15 with condylar position devices [Table 1]. The use of condylar positioning devices was avoided in

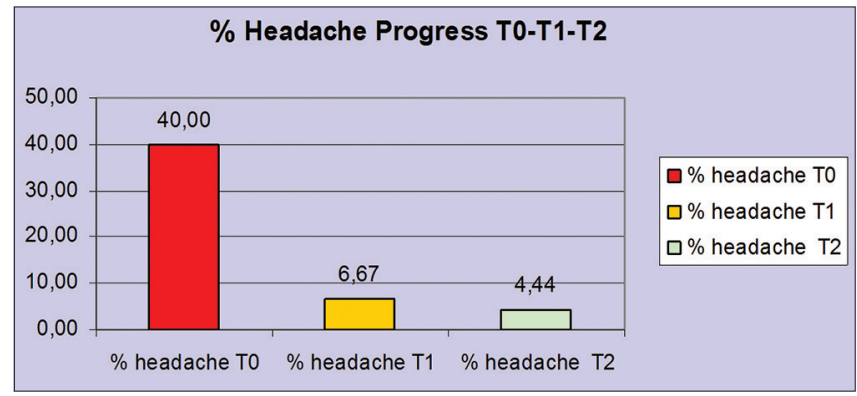

Figure 2: Headache progression during treatment 
patients with dentoskeletal malformations who exhibited no presurgical TMD. These devices are used in most patients who demonstrate more severe TMD prior to surgery. They are used during BSSO in therapeutic position with most patients having had a functional split therapy of TMJ prior to surgery.

\section{STATISTICAL ANALYSIS}

Results were evaluated with a paired-sample $t$-test and segmentation analysis. The paired-sample $t$-test procedure was used to test the hypothesis of no difference between the two variables. If the treatment had no effect, the average difference between the measurements was 0 . It is a "pre-post" design test. This method was used to analyze the mandibular functionality comparing the differences between the measurements of the mandibular movements.

The segmentation analysis evaluated the status of any patients (TMJ condyle disc coordination and arthralgia, muscular pain, headaches, and mandibular range of motions) prior to and after treatment. Data regarding each patient and the differences between their conditions pre and posttreatment were organized according to a depreciatory increasing alphanumeric scale ranging from 0 to 3 , where 0 corresponds to recovered/no symptoms, 1 corresponds to an improved condition (at least one symptom improved and no symptoms worsened), 2 corresponds to a stable status (no symptoms improved or worsened), and 3 corresponds to patient worsening (none symptoms improved and at least one worsen). A $P$ value of 0.05 was considered statistically significant.

\section{RESULTS}

During the study period, 45 patients were lost to followup, and hence the final sample consisted of 76 patients. Forty-seven patients $(61.8 \%)$ from the final study population were affected by TMD (female-to-male ratio, $3: 2$; mean age, 27 years). At the time of initial evaluation prior to orthodontic (presurgery) therapy (T0), 25 (33.3\%) patients experienced TMJ pain (arthralgia), 27 (35.5\%) had muscular pain (10 myofacial pain), 31 (40\%) suffered headaches (tension type and/or migraine), 42 (55.2\%) had disc dislocation with reduction, and 5 were affected by disc dislocation without reduction (6.5\%). Thirtyfive $(46.6 \%)$ patients had occlusal signs of parafunctions (bruxism and/or clenching), 8 (1.1\%) reported tinnitus, and $7(8.8 \%)$ dizziness.

The patients were evaluated again at the end of the orthodontic treatment immediately prior to the surgery. ${ }^{[16]}$ At this time, $21(27.6 \%)$ already exhibited signs and symptoms of TMDs: 17 (80.8\%) with arthralgia and disc dislocation with reduction and $4(19.2 \%)$ with muscular pain and limitation of mandibular movements.

\section{Discussion}

Before surgery, we performed a conservative therapy (splint therapy, physiotherapy, and pharmacotherapy tailored in various combination in respect of any singular patients) when TMJ and muscular signs and symptoms were present. This protocol was applied as the first therapy only when the disease was acute and the orthodontic treatment would be adversely affected. However, the conservative therapeutic protocol was always performed before surgery at the end of the orthodontic phase if articular and/or muscular signs and symptoms were present. In these patients, intraoperative condylar positioning devices were used. ${ }^{[17-20]}$

At T1, 3 months after the orthognathic surgery, TMJ pain changed from $33.3 \%$ (25 patients) to $4.44 \%$ (3 patients), muscular pain changed from $35.5 \%$ (27 patients) to $11.1 \%$ (8 patients), headaches improved from $40 \%$ (31 patients) to $6.67 \%$ (5 patients), and disc dislocation from $55.2 \%$ (42 patients) to $17.7 \%$ (13 patients). The most significant results can be seen from the comparison between $\mathrm{T} 0$ and $\mathrm{T} 2$ period [Table 2]. The improvement of arthralgia from $\mathrm{T} 1$ to $\mathrm{T} 2$ indicated no patients with this symptom at the final check-up. Headache symptoms, which were reported at $\mathrm{T} 2$, confirmed the same significant improvement obtained at $\mathrm{T} 1$; only $5(6.67 \%)$ patients were affected by this pathology after the treatment. Positive results for disc dislocation either

\begin{tabular}{lc}
\hline \multicolumn{2}{c}{ Table 1: Surgical treatment } \\
\hline $\begin{array}{l}\text { 12 Bilateral sagittal split osteotomy (6 with Condylar } \\
\text { Position Devices) } \\
\text { 64 Le Fort I + Bilateral sagittal split osteotomy (15 with }\end{array}$ & $84.2 \%$ \\
Condylar Position Devices) & \\
\hline
\end{tabular}

\begin{tabular}{lccc}
\hline \multicolumn{4}{c}{ Table 2: Symptoms time progress } \\
\hline Pain & T0 (\%) & T1 (\%) & T2 (\%) \\
Headache & 33.3 & 4.4 & 0 \\
Click & 40 & 6.6 & 4,4 \\
Bruxism & 62.2 & 17.7 & 13.3 \\
Clenching & 26.6 & 6.5 & 5.5 \\
Tinnitus & 20 & 6.5 & 7.5 \\
Dizzines & 1.1 & 0 & 0 \\
\% Change of & 8.8 & 0 & 0
\end{tabular}

$\%$ Change of symptoms between different periods of observation

\begin{tabular}{lc}
\hline \multicolumn{1}{c}{ Table 3: Segmentation analysis of TMDs } \\
\hline Patient Healed & $75.5 \%$ \\
Patient Improved & $17.9 \%$ \\
Patient Stable & $0 \%$ \\
Patient Worsening & $6.6 \%$ \\
\hline
\end{tabular}

At final examination (T2) more than $90 \%$ (71 patients) were considered completely recovered or improved 


\begin{tabular}{|c|c|c|c|c|c|c|}
\hline \multicolumn{7}{|c|}{ Table 4: Results of the paired sample $t$-test } \\
\hline & Medium & Standard Deviation & Medium bias & $t$ & df & Sig. (2-code) \\
\hline Couple 1 LatDXmm-0 - LatDXmm-3 & 1111 & 2,6412 & 3937 & 282 & 44 & 779 \\
\hline Couple 2 LatDXmm-0 - LatDXmm-6 & - & 2,9465 & 4392 & 2,227 & 44 & 028 \\
\hline Couple 3 LatDXmm-3 - LatDXmm-6 & 8889 & 1,7317 & 2581 & 3,443 & 44 & 001 \\
\hline Couple 4 LatSNmm-0 - LatSNmm-3 & 9000 & 2,9745 & 4434 & 2,030 & 44 & 048 \\
\hline Couple 5 LatSNmm-0 - LatDXmm-6 & 1000 & 3,0162 & 4496 & 222 & 44 & 825 \\
\hline Couple 6 LatSNmm -3--- LatSNmm -6 & - & 1,9738 & 2942 & 3,550 & 44 & 001 \\
\hline Couple 7 OpenMaxmm- 0 OpenMaxmm-3 & 7,9444 & 10,469 & 1,5607 & 5,090 & 44 & 000 \\
\hline Couple 8 OpenMaxmm-0 - OpenMaxmm-6 & 5,0898 & 8,5621 & 1,2764 & 3,988 & 44 & 000 \\
\hline Couple 9 OpenMaxmm-3 - OpenMaxmm-6 & - & 7,7591 & 1,1567 & 2,468 & 44 & 018 \\
\hline
\end{tabular}

"In red are highlighted the most statistically significant pairs

with or without reduction were also notable; from $61.8 \%$ (47 patients) at T0 to $13.3 \%$ (10 patients) at T2 with occasional disc dislocation with reduction.

In patients with and without TMDs, segmentation analysis highlighted the improvement caused about by the therapy; $57(75.5 \%)$ patients were considered to be recovered, $14(17.9 \%)$ improved, none were considered stable, whereas 5 patients $(6.6 \%)$ demonstrated some worsening in the form of TMJ sounds, 3 of these patients had not presented disc dislocation before surgery [Table 3]. At final examination (T2), more than $90 \%$ (71 patients) were considered completely recovered or improved.

The improvement of the mandibular functionality was determined by measuring the maximum mouth opening pre- and post-therapy. Before surgery, the minimum mouth opening was $24 \mathrm{~mm}$ and the maximum $64 \mathrm{~mm}$ with an average of $46.7 \mathrm{~mm}$ and a standard deviation of 8.8214 whereas at T2 the minimum was $36 \mathrm{~mm}$ and the maximum $54 \mathrm{~mm}$ with an average of $43.6 \pm 9.6582$.

Statistically significant results emerged from the paired sample t-test comparing the T0-T2 data of the mandibular lateral movements.

The most significant results can be observed on the pairsequences between the differences measurement of the mandibular range of motion. Hence, correlations between $\mathrm{T} 0-\mathrm{T} 1$ and between $\mathrm{T} 1-\mathrm{T} 2$ were statistically significant [Table 4].

\section{Conclusions}

We initiated this study to document the overall incidence of TMDs in patients with dentoskeletal deformities who were scheduled to undergo orthognathic surgery. The motivation for such documentation was to ascertain whether surgery could prove useful in the improvement or eradication of TMDs, in addition to correcting the structural deformities, as some data suggest. ${ }^{[21-24]}$ Our systematic collection of pre and postoperative data enabled us to make a proper evaluation of the possible benefits/detrimental effects of surgery on TMDs on this patient population.

TMDs in orthognathic population were present in $43.5 \%$ of the sample considered in our study; due to the prevalence of this pathology, a complete initial assessment was warranted. Only after such an assessment, it was possible to perform orthodontic, gnathologic, and surgical treatment in a balanced manner for any patient. ${ }^{[25-30]}$

Our data indicates beyond any doubt that both functional status and pain levels related to TMDs can be significantly improved with a multi-disciplinary approach. In addiction, we intend to highlight that the lateral mandibular movement slowly have improved, which is statistically significant. It should be also noted that it is possible to obtain these results only if the TMDs are properly assessed and resolved before surgical treatment.

We concluded that the surgeon's intervention need to be modified in the presence of presurgical TMDs. Further studies with larger patient populations, having the same skeletal malformation, are needed to confirm our hypothesis.

\section{FINANCIAL SUPPORT AND SPONSORSHIP}

Nil.

\section{CONFLICTS OF INTEREST}

There are no conflicts of interest.

\section{REFERENCES}

1. Forssell H, Kotiranta U, Kauko T, Suvinen T. Explanatory Models of Illness and Treatment Goals in Temporomandibular Disorder Pain Patients Reporting Different Levels of PainRelated Disability. J Oral Facial Pain Headache 2016;30:14-20.

2. Brauner E, Guarino G, Jamshir S, Papi P, Valentini V, Pompa V, et al. Evaluation of Highly Porous Dental Implants in Postablative Oral and Maxillofacial Cancer Patients: A Prospective Pilot Clinical Case Series Report. Implant Dent 2015;24:631-7.

3. Pompa V, Valentini V, Pompa G, Di Carlo S, Bresadola L. Treatment of high-flow arteriovenous malformations (AVMs) of 
the head and neck with embolization and surgical resection. Ann Ital Chir 2011;82:253-9.

4. Conti PC, Costa YM, Gonçalves DA, Svensson P. Headaches and myofascial temporomandibular disorders: Overlapping entities, separate managements? J Oral Rehabil 2016;43:702-15.

5. Antunes Ortega AC, Pozza DH, Rocha Rodrigues LL, Guimarães AS. Relationship Between Orthodontics and Temporomandibular Disorders: A Prospective Study. J Oral Facial Pain Headache 2016;30:134-8.

6. Cascone $\mathrm{P}$, Di Paolo $\mathrm{C}$, Leonardi R, Pedullà E. Temporomandibular disorders and orthognathic surgery. J Craniofac Surg 2008;19:687-92.

7. Campi LB, Jordani PC, Tenan HL, Camparis CM, Gonçalves DA. Painful temporomandibular disorders and central sensitization: Implications for management-a pilot study. Int J Oral Maxillofac Surg 2016;16:30147-9.

8. Graff-Radford SB, Abbott JJ. Temporomandibular Disorders and Headache. Oral Maxillofac Surg Clin North Am 2016;28:335-49.

9. Pakula K, Somppi M, Finne K, Oikarinen K. Effects of orthognathic surgery on temporomandibular joint dysfunction. A controlled prospective 4-year follow-up study. Int J Oral Maxillofac Surg 2000;29:183-7.

10. Dervis E, Tuncer E. Long-term evaluations of temporomandibular disorders in patients undergoing orthognathic surgery compared with a control group. Oral Surg Oral Med Oral Pathol Oral Radiol Endod 2002;94:554-60.

11. Wolford LM, Reiche-Fischel O, Mehra P. Changes in temporomandibular joint dysfunction after orthognathic surgery. J Oral Maxillofac Surg 2003;61:655-60.

12. Aoyama S, Kino K, Kobayashi J, Yoshimasu H, Amagasa T. Clinical evaluation of the temporomandibular joint following orthognathic surgery-multiple logistic regression analysis. J Med Dent Sci 2005;52:109-14.

13. Abrahamsson C, Ekberg E, Henrikson T, Bondemark L. Alterations of temporomandibular disorders before and after orthognathic surgery: A systematic review. Angle Orthod 2007;77:729-34.

14. Abrahamsson C, Ekberg E, Henrikson T, Nilner M, Sunzel B, Bondemark L. TMD in consecutive patients referred for orthognathic surgery. Angle Orthod 2009;79:621-7.

15. Westermark A, Shayeghi F, Thor A. Temporomandibular dysfunction in 1,516 patients before and after orthognathic surgery. Int J Adult Orthodon Orthognath Surg 2001;16:145-51.

16. Costa YM, Porporatti AL, Stuginski-Barbosa J, Bonjardim LR, Speciali JG, Rodrigues Conti PC. Headache Attributed to Masticatory Myofascial Pain: Clinical Features and Management Outcomes. J Oral Facial Pain Headache 2015;29:323-30.
17. Silvola AS, Tolvanen M, Rusanen J, Sipilä K, Lahti S, Pirttiniemi P. Do changes in oral health-related quality-of-life, facial pain and temporomandibular disorders correlate after treatment of severe malocclusion? Acta Odontol Scand 2016;74:44-50.

18. Scolozzi P, Wandeler PA, Courvoisier DS. Can clinical factors predict postoperative temporomandibular disorders in orthognathic patients? A retrospective study of 219 patients. Oral Surg Oral Med Oral Pathol Oral Radiol 2015;119:531-8.

19. Nale JC. Orthognathic surgery and the temporomandibular joint patient. Oral Maxillofac Surg Clin North Am 2014;26:551-64.

20. Gil-Martínez A, Grande-Alonso M, López-de-UraldeVillanueva I, López-López A, Fernández-Carnero J, La Touche R. Chronic Temporomandibular Disorders: Disability, pain intensity and fear of movement. J Headache Pain 2016;17:103.

21. Mazzone N, Matteini C, Incisivo V, Belli E. Temporomandibular joint disorders and maxillomandibular malformations: Role of condylar "reposition in" plate. J Craniofac Surg 2009;20:909-15.

22. Costa F, Robiony M, Toro C, Sembronio S, Polini F, Politi M. Condylar positioning devices for orthognathic surgery: A literature review. Oral Surg Oral Med Oral Pathol Oral Radiol Endod 2008;106:179-90.

23. Pahkala R, Heino J. Effects of sagittal split ramus osteotomy on temporomandibular disorders in seventy-two patients. Acta Odontol Scand 2004;62:238-44.

24. Onizawa K, Schmelzeisen R, Vogt S. Alteration of temporomandibular joint symptoms after orthognathic surgery: Comparison with healthy volunteers. J Oral Maxillofac Surg 1995;53:117-21

25. Magnusson T, Ahlborg G, Finne K, Nethander G, Svartz K. Changes in temporomandibular joint pain-dysfunction after surgical correction of dentofacial anomalies. Int $\mathrm{J}$ Oral Maxillofac Surg 1986;15:707-14.

26. Kalha A. Orthognathic treatment and temporomandibular disorders - part 1. Evid Based Dent 2010;11:82-3.

27. Lindenmeyer A, Sutcliffe $P$, Eghtessad $M$, Goulden $R$, Speculand B, Harris M. Oral and maxillofacial surgery and chronic painful temporomandibular disorders - A systematic review. J Oral Maxillofac Surg 2010;68:2755-64.

28. D'Urso A, Serritella E, Tolevski Meshkova D, Falisi G, Di Paolo C. Headache and temporo mandibular disorders: Epidemiological assessment. Minerva Stomatol 2016;65:85-92.

29. Attanasio G, Leonardi A, Arangio P, Minni A, Covelli E, Pucci R, et al. Tinnitus in patients with temporo-mandibular joint disorder: Proposal for a new treatment protocol. J Craniomaxillofac Surg 2015;43:724-7.

30. Cassetta M, Pranno N, Pompa V, Barchetti F, Pompa G. High resolution 3-T MR imaging in the evaluation of the trigeminal nerve course. Eur Rev Med Pharmacol Sci 2014;18:257-64. 